\title{
Severe Rhabdomyolysis and Acute Renal Failure Secondary to Concomitant Use of Simvastatin, Amiodarone, and Atazanavir
}

\author{
Ginelle A. Schmidt, PharmD, James D. Hoehns, PharmD, BCPS, \\ Jessica L. Purcell, PharmD, MPH, Robert L. Friedman, MD, and Yasir Elhawi, MD
}

Objective: To report a case of a severe interaction between simvastatin, amiodarone, and atazanavir resulting in rhabdomyolysis and acute renal failure.

Background: A 72-year-old white man with underlying human immunodeficiency virus, atrial fibrillation, coronary artery disease, and hyperlipidemia presented with generalized pain, fatigue, and dark orange urine for 3 days. The patient was taking $80 \mathrm{mg}$ simvastatin at bedtime (initiated 27 days earlier); amiodarone at a dose of $400 \mathrm{mg}$ daily for 7 days, then $200 \mathrm{mg}$ daily (initiated 19 days earlier); and 400 mg atazanavir daily (initiated at least 2 years previously). Laboratory evaluation revealed 66,680 U/L creatine kinase, $93 \mathrm{mg} / \mathrm{dL}$ blood urea nitrogen, $4.6 \mathrm{mg} / \mathrm{dL}$ creatinine, $1579 \mathrm{U} / \mathrm{L}$ aspartate aminotransferase, and $738 \mathrm{U} / \mathrm{L}$ alanine aminotransferase. Simvastatin, amiodarone, and the patient's human immunodeficiency virus medications were all temporarily discontinued and the patient was given forced alkaline diuresis and started on dialysis. Nine days later the patient's creatine kinase had dropped to $1695 \mathrm{U} / \mathrm{L}$ and creatinine was $3.3 \mathrm{mg} / \mathrm{dL}$. The patient was discharged and continued outpatient dialysis for 1 month until his renal function recovered.

Discussion: The risk of rhabdomyolysis is increased in the presence of concomitant drugs that inhibit simvastatin metabolism. Simvastatin is metabolized by CYP3A4. Amiodarone and atazanavir are recognized CYP3A4 inhibitors.

Conclusions: Pharmacokinetic differences in statins are an important consideration for assessing the risk of potential drug interactions. In patients requiring the concurrent use of statins and CYP3A4 inhibitors, pravastatin, fluvastatin, and rosuvastatin carry the lowest risk of drug interactions; atorvastatin carries moderate risk, whereas simvastatin and lovastatin have the highest risk and should be avoided in patients taking concomitant CYP3A4 inhibitors. (J Am Board Fam Med 2007;20:411-416.)

The ability of statins to reduce the risk of cardiovascular morbidity and mortality in patients with dyslipidemia is well established. ${ }^{1-3}$ In addition to their unmatched efficacy, the adverse effects associated with statins are usually mild and transient. ${ }^{4}$ The most noteworthy adverse effects associated with statins are elevations in liver transaminases,

This article was externally peer reviewed.

Submitted 10 October 2006; revised 2 February 2007; accepted 12 February 2007.

From Department of Pharmacy, University of Iowa College of Pharmacy, Iowa City (GAS, JDH); and the Northeast Iowa Family Practice Center, Waterloo (JDH, JLP, RLF, YE).

Funding: none.

Conflict of interest: none declared.

Corresponding author: James D. Hoehns, PharmD, BCPS, University of Iowa College of Pharmacy/Northeast Iowa Family Practice Center, 2055 Kimball Avenue, Waterloo, IA 50702 (E-mail: jhoehns@neimef.org). myopathy, and rhabdomyolysis, which is characterized by massive muscle necrosis, myoglobinuria, and acute renal failure. ${ }^{5,6}$ The risk of rhabdomyolysis with statin monotherapy is low and dose related. ${ }^{4,7-10}$ This risk increases in patients taking concomitant drugs that inhibit the cytochrome P450-related statin metabolism such as azole antifungals, cyclosporine, fibrates, macrolides, and non-dihydropyridine calcium channel blockers. ${ }^{4,7,8}$ Patients with coronary artery disease are often on a number of medications and therefore the potential for drug interactions is an important consideration. This article describes a case of rhabdomyolysis in a patient taking concomitant simvastatin, amiodarone, and atazanavir.

\section{Case Report}

A 72-year-old white man (weight, $72 \mathrm{~kg}$; height, $175 \mathrm{~cm}$; body mass index, $23.5 \mathrm{~kg} / \mathrm{m}^{2}$ ) presented to 
the hospital emergency department with complaints of generalized muscle pain (described as dull and constant) that persisted for 4 days, weakness, fatigue, decreased appetite, and dark orange urine for 3 days. Physical examination showed a pale, seemingly ill elderly man, but was otherwise largely unremarkable. His medical history included human immunodeficiency virus (HIV), hyperlipidemia, benign prostatic hyperplasia, chronic obstructive pulmonary disease, hypertension, atrial fibrillation, pacemaker insertion (6 months earlier), and coronary artery disease with myocardial infarction (3 years earlier). The patient had no history of renal disease, with a baseline creatinine of $0.9 \mathrm{mg} / \mathrm{dL} .80$ $\mathrm{mg}$ simvastatin daily was initiated 27 days earlier; before this he had taken $40 \mathrm{mg}$ atorvastatin daily for 2 years without any adverse effects. He began taking amiodarone 19 days before hospitalization at a dose of $400 \mathrm{mg}$ daily for 7 days, then $200 \mathrm{mg}$ daily. Additional chronic medications included his HIV medications (400 mg atazanavir at bedtime [protease inhibitor], $40 \mathrm{mg}$ stavudine twice a day [nucleoside reverse transcriptase inhibitor], and $600 \mathrm{mg}$ delavirdine twice a day [non-nucleoside reverse transcriptase inhibitor]), all which he had taken for 2 years. In addition, the patient took isosorbide mononitrate, $60 \mathrm{mg}$ twice a day; clopidogrel, $75 \mathrm{mg}$ daily; aspirin, $325 \mathrm{mg}$ daily; metoprolol, $50 \mathrm{mg}$ twice a day; terazosin, $5 \mathrm{mg}$ at bedtime; famotidine, $20 \mathrm{mg}$ twice a day; polyethylene glycol, $17 \mathrm{~g}$ at bedtime; and docusate, $200 \mathrm{mg}$ daily.

The patient's vital signs were temperature, $37.4^{\circ} \mathrm{C}$; pulse, 50 beats per minute and paced; blood pressure, 101/30; and respirations, 18 breaths per minute. Laboratory evaluation at admission revealed a creatine kinase of $66,680 \mathrm{U} / \mathrm{L}$; aspartate aminotransferase of $1,579 \mathrm{U} / \mathrm{L}$; alanine aminotransferase of $738 \mathrm{U} / \mathrm{L}$; blood urea nitrogen of $90 \mathrm{mg} / \mathrm{dL}$; creatinine of $4.1 \mathrm{mg} / \mathrm{dL}$; serum potassium of $6.7 \mathrm{mEq} / \mathrm{L}$; and thyroid stimulating hormone of $9.4 \mathrm{U} / \mathrm{L}$.

The patient was diagnosed with rhabdomyolysis and oliguric acute renal failure. Simvastatin, amiodarone, and his HIV medications were all temporarily discontinued. He was given forced alkaline diuresis (intravenous normal saline with bicarbonate) and started on dialysis on day 2 . The creatine kinase level peaked at $>70,000 \mathrm{U} / \mathrm{L}$ (upper limit of assay) on days 4 and 5 of admission. At discharge (9 days after hospitalization), laboratory test results were creatine kinase level of $1695 \mathrm{U} / \mathrm{L}$ and a cre- atinine level of $3.3 \mathrm{mg} / \mathrm{dL}$. The patient was restarted on amiodarone at this time and continued outpatient dialysis 3 times a week for 1 month, after which his creatinine level had reduced to $1.6 \mathrm{mg} /$ $\mathrm{dL}$, his aspartate aminotransferase level reduced to $30 \mathrm{U} / \mathrm{L}$, and his alanine aminotransferase reduced to $22 \mathrm{U} / \mathrm{L}$. The patient was restarted on his HIV medications 6 weeks after hospitalization; however, no statins were restarted because of his history of rhabdomyolysis. The patient reported some mild residual weakness until 1 month after hospitalization, but has otherwise made a full recovery.

\section{Discussion}

According to the research firm IMS Health, statins represent the top drug class in the United States in sales; atorvastatin and simvastatin were the first and second most prescribed statins in 2004, respectively. ${ }^{11}$ Given their widespread use and because several agents in this class share a metabolic pathway common to many other drugs, it is important to recognize the potential for drug-drug interactions with statins, which may increase the potential for rhabdomyolysis.

The reported risk of rhabdomyolysis with simvastatin monotherapy is low and dose related: $0.02 \%$ at $20 \mathrm{mg}$ daily, $0.07 \%$ at $40 \mathrm{mg}$ daily, and $0.3 \%$ at $80 \mathrm{mg}$ daily. ${ }^{5}$ Several factors have been identified that increase the risk for both myopathy and rhabdomyolysis, including advanced age, chronic renal insufficiency, metabolic disorders such as diabetes or hypothyroidism, major surgery, and alcohol abuse. ${ }^{4,5,7,12,13}$ In addition, the manufacturer of simvastatin has identified higher simvastatin doses and the use of certain other medications as risk factors. ${ }^{14}$ These medications include azole antifungals, macrolide antibiotics, HIV protease inhibitors, nefazodone, cyclosporine, danazol, and verapamil. ${ }^{13,14}$ Grapefruit juice also has the potential to interact with simvastatin; therefore, the manufacturer cautions patients not to consume large quantities ( $>1$ quart) of grapefruit juice while taking simvastatin. ${ }^{14}$ In May 2002, the manufacturer of simvastatin revised its product information to include warnings against using simvastatin doses more than $20 \mathrm{mg}$ in combination with amiodarone. ${ }^{14}$ According to data after marketing, $6 \%$ of patients taking amiodarone and $80-\mathrm{mg}$ simvastatin in combination reported myopathy. ${ }^{14,15}$ This patient's risk factors included advanced age and con- 
Table 1. Summary of Published Case Reports Describing an Interaction Between Simvastatin and Miodarone

\begin{tabular}{|c|c|c|c|c|c|c|}
\hline Details & Patient $1^{7}$ & Patient $2^{8}$ & Patient $3^{12}$ & Patient $4^{16}$ & Patient $5^{21}$ & Current Patient \\
\hline Age/sex & $72 /$ male & $63 /$ male & 77/male & $68 /$ female & $56 /$ male & $72 /$ male \\
\hline Simvastatin dose* & $80 \mathrm{mg}$ & $40 \mathrm{mg}$ & $80 \mathrm{mg}$ & $40 \mathrm{mg}$ & $40 \mathrm{mg}$ & $80 \mathrm{mg}$ \\
\hline Amiodarone dose* & $200 \mathrm{mg}$ & $200 \mathrm{mg}$ & $100 \mathrm{mg}$ & Not reported & $600 \mathrm{mg}$ & $200 \mathrm{mg}$ \\
\hline $\begin{array}{c}\text { Other risk } \\
\text { factors } \dagger\end{array}$ & $\begin{array}{l}\text { Chronic renal } \\
\text { insufficiency, age, } \\
\text { diabetes, recent } \\
\text { surgery }\end{array}$ & $\begin{array}{l}\text { Age, diabetes, } \\
\text { recent surgery }\end{array}$ & $\begin{array}{l}\text { Chronic renal } \\
\text { insufficiency, age, } \\
\text { hypo-thyroidism }\end{array}$ & $\begin{array}{l}\text { Age, } \\
\text { verapamil }\end{array}$ & $\begin{array}{l}\text { Recent surgery, } \\
\text { clarithromycin }\end{array}$ & $\begin{array}{l}\text { Age, atazanavir, } \\
\text { delviradine }\end{array}$ \\
\hline $\begin{array}{l}\text { Reported adverse } \\
\text { event }\end{array}$ & Rhabdomyolysis & Rhabdomyolysis & Rhabdomyolysis & Myopathy & Myositis & Rhabdomyolysis \\
\hline $\begin{array}{l}\text { Time to adverse } \\
\text { event } \ddagger\end{array}$ & 39 days & 14 days & 21 days & 2 years & 1 to 9 days & 19 days \\
\hline $\begin{array}{l}\text { Peak creatine } \\
\text { kinase }(\mathrm{U} / \mathrm{L})\end{array}$ & 19,620 & 40,392 & 28,523 & 48,000 & $>20,000$ & $>70,000$ \\
\hline Dialysis required & No & No & No & No & No & Yes \\
\hline Outcome & Resolved & Resolved & Resolved & Resolved & Resolved & $\begin{array}{l}\text { Resolved after } 1 \\
\text { month of } \\
\text { dialysis }\end{array}$ \\
\hline
\end{tabular}

*Dose at time of adverse event.

tOther risk factors include advanced age, chronic renal insufficiency, metabolic disorders such as diabetes or hypothyroidism, major surgery, alcohol abuse, and the use of concomitant medications (azole antifungals, macrolide antibiotics, HIV protease inhibitors, nefazodone, cyclosporine, danazol, verapamil, and amiodarone).

$\neq$ Time to adverse event from the time that amiodarone and simvastatin were being administered concomitantly.

comitant medications, particularly amiodarone and his protease inhibitor, atazanavir.

Important pharmacokinetic differences exist between the available statins (atorvastatin, fluvastatin, lovastatin, rosuvastatin, pravastatin, simvastatin). Although pravastatin is primarily eliminated by sulfation, all other available statins are metabolized by the cytochrome $\mathrm{P} 450$ system. $^{4}$ Fluvastatin and rosuvastatin are primarily metabolized by CYP2C9. ${ }^{4}$ The remaining 3 statins-atorvastatin, lovastatin, and simvastatin-all undergo extensive first-pass metabolism by CYP3A4, resulting in an absolute oral bioavailability of $12 \%, 20 \%$, and $5 \%$, respectively. ${ }^{4,5}$ CYP3A4 is one of the most important drug metabolizing enzymes and is responsible for the oxidative metabolism of more than $50 \%$ of drugs used in humans. ${ }^{5,16}$ Agents such as these, with low bioavailability and primary dependence on CYP3A4 for elimination, are susceptible to changes in their pharmacokinetics when administered with a $3 \mathrm{~A} 4$ inhibitor. ${ }^{17}$ Simvastatin and lovastatin are administered as prodrugs that are hydrolyzed to the active $\beta$-hydroxyacid form. ${ }^{5}$ In contrast, atorvastatin is administered in its active form and has CYP3A-mediated active metabolites that are responsible for nearly $70 \%$ of its activity. ${ }^{5,18}$ Inhibition of first-pass metabolism of any of these 3 statins could potentially result in increased circu- lating levels and risk of toxicity. This is particularly true for simvastatin and lovastatin, in which steadystate concentrations may be increased by 10 - to 20-fold. ${ }^{5}$ Inhibition of atorvastatin metabolism, however, is likely to produce a more balanced effect with a smaller amount of drug being converted to its active metabolites. ${ }^{5}$

Amiodarone is a potent inhibitor of CYP3 A4 $4{ }^{4,5}$ This inhibition is primarily because of its active metabolite, desethylamiodarone, which noncompetitively inhibits $3 \mathrm{~A} 4 .{ }^{19}$ The proposed mechanism to explain a drug interaction between amiodarone and simvastatin is a decrease in simvastatin metabolism because of inhibition by amiodarone. Use of the Naranjo adverse drug event probability scale indicated that the rhabdomyolysis experienced in our reported case was "probably" the result of an interaction between simvastatin and amiodarone/atazanavir. $^{20}$ In previously reported cases, amiodarone has been found to increase rhabdomyolysis risk when taken with simvastatin (Table 1). $7,8,12,16,21$

The use of lipid-lowering therapy in patients with HIV is becoming increasingly more common. This is in part because of the increasing life expectancy of patients with HIV/AIDS; in addition, several agents used to treat HIV can cause increases in serum lipids. ${ }^{22,23}$ Specifically, certain agents of the HIV protease inhibitor class, including ritonavir, 
Table 2. Summary of Manufacturer Recommendations for Statin and Protease Inhibitor Drug Interactions as Described in Prescribing Information

\begin{tabular}{|c|c|c|c|c|c|c|}
\hline $\begin{array}{l}\text { CYP3A4 } \\
\text { Inhibitor }\end{array}$ & Simvastatin & Lovastatin & Atorvastatin & Pravastatin & Fluvastatin & Rosuvastatin \\
\hline Atazanavir & Not recommended & Not recommended & Use with caution & $\begin{array}{l}\text { Clinically significant } \\
\text { DIs are not } \\
\text { expected }\end{array}$ & $\begin{array}{l}\text { Clinically significant } \\
\text { DIs are not } \\
\text { expected }\end{array}$ & N/A \\
\hline Nelfinavir & Not recommended* & Not recommended & Use with caution & Considered safe & Considered safe & N/A \\
\hline Ritonavir & Not recommended ${ }^{*}$ & Not recommended & Use with caution & Considered safe & Considered safe & N/A \\
\hline Indinavir & Not recommended & Not recommended & Use with caution & Considered safe & Considered safe & Considered safe \\
\hline Saquinavir & Not recommended & Not recommended & Use with caution & Considered safe & Considered safe & Considered safe \\
\hline Amprenavir & Not recommended & Not recommended & Use with caution & Considered safe & Considered safe & N/A \\
\hline Fosamprenavir & Not recommended & Not recommended & Use with caution & Considered safe & Considered safe & Considered safe \\
\hline $\begin{array}{l}\text { Lopinavir/ } \\
\text { ritonavir }\end{array}$ & Not recommended & Not recommended & Use with caution* & Considered safe & Considered safe & N/A \\
\hline Tipranavir & Not recommended & Not recommended & Use with caution & N/A & N/A & N/A \\
\hline
\end{tabular}

${ }^{*}$ Rhabdomyolysis has been described. ${ }^{26,28-30}$

DI, drug interaction; N/A, interaction not reported.

indinavir, and nelfinavir, can induce elevations in serum lipids. ${ }^{23}$ Cytochrome P4503A isoenzymes are responsible for the metabolism of all currently available HIV protease inhibitors, which are both metabolized by and are inhibitors of CYP3A. ${ }^{22,24-26}$ Therefore, a significant potential exists for drug-drug interactions with the combination of protease inhibitors and statins via competitive and reversible inhibition of CYP3A4. ${ }^{17,24}$

The use of atazanavir and simvastatin in combination has not been studied to date. ${ }^{25}$ There are, however, a number of reports describing the effect of concomitant use of statins and other protease inhibitors. $^{22,24,26-32}$ One such study looked at the coadministration of ritonavir plus saquinavir (both protease inhibitors) and their effect on the pharmacokinetics of pravastatin, simvastatin, and atorvastatin, each at a dose of $40 \mathrm{mg}$ daily. ${ }^{24}$ They observed a decline in pravastatin area under the curve $(P=.005)$, whereas atorvastatin, total active atorvastatin, and simvastatin areas under the curve all increased significantly $(P<.001)$ in combination with ritonavir/saquinavir. ${ }^{24}$ The elevations in the area under the curve of atorvastatin was 1.7 times that before the addition of the ritonavir/saquinavir, whereas the elevation in the area under the curve of simvastatin was more than 30 -fold. ${ }^{24}$ There were no cases of rhabdomyolysis in this study. The authors concluded that pravastatin seems to be safe and atorvastatin can probably be used with caution in patients taking ritonavir/saquinavir, but use of simvastatin should be avoided. ${ }^{24}$ Hare et $\mathrm{al}^{26}$ described a case of rhabdomyolysis and death of a patient using combination $80 \mathrm{mg}$ simvastatin and nelfinavir, another protease inhibitor. Hsyu et $\mathrm{al}^{22}$ reported increases in simvastatin $(20 \mathrm{mg})$ concentrations of more than $500 \%$ when combined with nelfinavir, and only moderate increased concentrations of atorvastatin $(10 \mathrm{mg})$ between $70 \%$ and $120 \%$. This demonstrates that simvastatin is more susceptible to drug-drug interactions with protease inhibitors when compared with atorvastatin.

Although all currently marketed protease inhibitors interfere with CYP3A activity, each does so to varying degrees..$^{23,27,29,33}$ Ritonavir has been recognized as having the highest potential for such interactions. ${ }^{26,27}$ This is followed by indinavir, nelfinavir, amprenavir, and saquinavir, in decreasing order..$^{22,23,33}$ It is unclear where atazanavir fits into this scale. Because of its unique elimination pathway, pravastatin is considered the statin of choice for patients taking concomitant protease inhibitors. ${ }^{29}$ Alternatives to pravastatin include atorvastatin, administered at a starting dose of $10 \mathrm{mg}$ daily with close monitoring, or fluvastatin, which is primarily metabolized by CYP2C $9 .{ }^{23}$ Major drug interactions are most likely to occur with simvastatin and lovastatin; therefore, these 2 agents should be avoided in this patient population (Table 2). ${ }^{23}$

Pharmacokinetic differences in the various available statins are an important consideration for drug selection. Clinicians usually focus on achieving lipid goals during patient care and subsequently may not consider the differences in drug-drug interaction potential among the statins when selecting an agent. This is of particular concern with the recent release of generic simvastatin and, consequently, many changes in insurance formularies. 
Patients are often switched between statins for insurance purposes (as was the case with our patient) without full consideration of the implications of pharmacokinetic differences. Our patient had previously been taking $40 \mathrm{mg}$ atorvastatin once daily in addition to his HIV medications for more than 2 years without adverse effects. In addition to considering pharmacokinetic differences when initiating a statin, all patients should be educated on the signs and symptoms of rhabdomyolysis, including muscle pain, weakness, and darkened urine. In patients at hightened risk of rhabomyolysis, it is reasonable to consider measuring a baseline creatine kinase level and obtain a second measurement in all patients experiencing such signs or symptoms to determine the severity of muscle toxicity.

We reported above about a patient who suffered rhabdomyolysis and acute renal failure, most likely secondary to a drug interaction between simvastatin, amiodarone, and atazanavir. This case is notable because the patient experienced the highest elevations in creatinine kinase levels, 2 to 3 times that of any similar reported cases involving amiodarone- and simvastatin-related rhabdomyolysis to date,,$^{7,8,12}$ and required nearly 1 month of dialysis before his kidney function approached baseline functioning. Although amiodarone has been previously reported to increase the risk of rhabdomyolysis when taken concomitantly with simvastatin, we believe the severity reported in our case may also be caused in part by the concomitant use of atazanavir. This is the first reported case of rhabdomyolysis in which atazanavir use may have contributed to the decreased metabolism of simvastatin.

Interpretation of this case is limited by several factors. One limitation is distinguishing between the contributing etiology of amiodarone and atazanavir. Although the patient had previously tolerated $40 \mathrm{mg}$ atorvastatin, the time interval between when the patient was switched to simvastatin and amiodarone was initiated was not long enough to determine whether the adverse reaction was primarily a result of the addition of amiodarone or if it was caused by the pharmacokinetic differences between atorvastatin and simvastatin in terms of interaction with the patient's HIV medications. Another limitation of our report is the additional factors that may have contributed to the severity of the interaction experienced. The patient was taking delavirdine, a non-nucleoside reverse transcriptase inhibitor, which is an inhibitor of CYP3A4 and therefore has the potential to interact with simvastatin as well. ${ }^{23,31,33}$ This drug-drug interaction, however, has not been well established. A Medline search revealed only one report identifying a possible drug interaction between delavirdine and atorvastatin $20 \mathrm{mg}$ daily, resulting in rhabdomyolysis and acute renal failure. ${ }^{31}$ Finally, there have been a few cases reported identifying hypothyroidism as a cause of rhabdomyolysis. ${ }^{34,35}$ In most of the cases reported, however, there has been a precipitating factor such as exercise or chronic renal failure; very few cases have been attributed to hypothyroidism alone. ${ }^{34}$ Although our patient presented with a mild elevation in thyrotropin, this was not believed to have contributed to his rhabdomyolysis. Rather, this elevation could likely be caused by amiodarone inhibiting the peripheral conversion of thyroxine (T4) to triiodothyronine (T3). ${ }^{36}$

\section{Conclusion}

Simvastatin is largely dependent on the CYP3A4 enzyme system for its metabolism and it interacts with many commonly prescribed drugs. In patients requiring the concurrent use of statins and CYP3A4 inhibitors, pravastatin or fluvastatin would be reasonable choices to minimize potential drug-drug interactions, whereas atorvastatin seems to carry moderate risk and should be used with caution. Simvastatin and lovastatin carry the highest risk of drug interactions and should be avoided in patients taking concomitant CYP3A4 inhibitors such as amiodarone and protease inhibitors.

\section{References}

1. Randomized trial of cholesterol lowering in 4444 patients with coronary heart disease: the Scandinavian Simvastatin Survival Study (4S). Lancet 1994; 344:1383-9.

2. Heart Protection Study Collaborative Group. MRC/ BHF Heart Protection Study of cholesterol lowering with simvastatin in 20,536 high-risk individuals: a randomised placebo-controlled trial. Lancet 2002; 360:7-22.

3. Prevention of cardiovascular events and death with pravastatin in patients with coronary heart disease and a broad range of initial cholesterol levels. The Long-term Intervention with Pravastatin in Ischemic Disease (LIPID) Study Group. N Engl J Med 1998;339:1349-57.

4. Bellosta S, Paoletti R, Corsini A. Safety of statins: focus on clinical pharmacokinetics and drug interactions. Circulation 2004;109:50-7. 
5. Williams D, Freely J. Pharmacokinetic-pharmacodynamic drug interactions with HMG-CoA reductase inhibitors. Clin Pharmacokinet 2002;41:343-70.

6. Pasternak RC, Smith SC Jr, Bairey-Merz CN, et al. ACC/AHA/NHLBI clinical advisory on the use and safety of statins. J Am Coll Cardiol 2002;40:567-72.

7. Ricaurte B, Guirguis A, Taylor HC, Zabriskie D. Simvastatin-amiodarone interaction resulting in rhabdomyolysis, azotemia, and possible hepatotoxicity. Ann Pharmacother 2006;40:753-7.

8. Wratchford P, Ponte CD. High-dose simvastatin and rhabdomyolysis. Am J Health Syst Pharm 2003; 60:698-700.

9. Graham D, Staffa J, Shatin D, et al. Incidence of hospitalized rhabdomyolysis in patients treated with lipid-lowering drugs. JAMA 2004;292:2585-90.

10. Jacobson T. Comparative pharmacokinetic interaction profiles of pravastatin, simvastatin, and atorvastatin when coadministered with cytochrome P450 inhibitors. Am J Cardiology 2004;94:1140-46.

11. IMS Health. Press room. 2004 year-end U.S. prescription and sales information and commentary. IMS Health Incorporated; 2007 [cited 2005 February]. Available from: http://www.imshealth.com/ims/portal/ front/articleC/0,2777,6599_3665_69890098,00.html.

12. Roten L, Schoenenberger RA, Krahenbuhl S, Schlienger R. Rhabdomyolysis in association with simvastatin and amiodarone. Ann Pharmacother 2004;38:978-81.

13. Grundy S. The issue of statin safety: Where do we stand? Circulation 2005;111:3016-19.

14. Zocor (simvastatin) package insert. Whitehouse Station (NJ): Merck \& Co. Inc.; 2004.

15. Alsheikh-Ali A, Karas RH. Adverse events with concomitant amiodarone and statin therapy. Prev Cardiol 2005;8:95-7.

16. McKelvie PA, Dennett X. Myopathy associated with HMG-CoA reductase inhibitors (statins): A series of 10 patients and review of literature. J Clin Neuro Disease 2003;3:143-8.

17. Dresser G, Spence D, Bailey D. Pharmacokineticpharmacodynamic consequences and clinical relevance of cytochrome P450 3A4 inhibition. Clin Pharmacokint 2000;38:41-57.

18. Lipitor (atorvastatin) prescribing information. Pfizer Inc.; 2005.

19. Ohyama K, Nakajima M, Suzuki M, Shimada N, Yamazaki H, Yokoi T. Inhibitory effects of amiodarone and its $\mathrm{N}$-desethylated metabolite on human cytochrome P450 activities: prediction of in vivo drug interactions. Br J Clin Pharmacol 2000;49:244-53.

20. Naranjo CA, Busto U, Sellers EM, et al. A method for estimating the probability of adverse reactions. Clin Pharmacol Ther 1981;30:239-45.

21. Chouhan UM, Chakrabarti S, Millward LJ. Simva- statin interaction with clarithromycin and amiodarone causing myositis. Ann Pharmacother 2005;39: 1760-1; Epub 2005 Sept 13.

22. Hsyu PH, Schultz-Smith MD, Lillibridge JH, Lewis RH, Kerr BM. Pharmacokinetic interactions between nelfinavir and 3-hydroxy-3-methylglutaryl coenzyme A reductase inhibitors atorvastatin and simvastatin. Antimicrob Agents Chemother 2001;4: 3445-50.

23. Sax PE. Strategies for management and treatment of dyslipidemia in HIV/AIDS. AIDS Care 2006; 18:149-57.

24. Fichtenbaum C, Gerber J, Rosenkranz S, et al. Pharmacokinetic interactions between protease inhibitors and statins in HIV seronegative volunteers: ACTG Study A5047. AIDS 2002;16:569-77.

25. Reyataz (atazanavir sulfate) package insert. BristolMyers Squibb Company; 2006.

26. Hare CB, Vu MP, Grunfeld C, Lampiris HW. Simvastatin-nelfinavir interaction implicated in rhabdomyolysis and death. CID 35:e111-e112, 2002.

27. Bonaventura C, Negredo E. HIV protease inhibitors and dyslipidemia. AIDS Rev 2003;5:19-24.

28. Moro H, Tsukada H, Tanuma A, et al. Rhabdomyolysis after simvastatin therapy in an HIV-infected patient with chronic renal failure. AIDS Patient Care STDS 2004;18:687-90.

29. Cheng CH, Miller C, Lowe C, Pearson VE. Rhabdomyolysis due to probable interaction between simvastatin and ritonavir. Am J Health Syst Pharm 2002; 59:728-30.

30. Mah Ming JB, Gill MJ. Drug-induced rhabdomyolysis after concomitant use of clarithromycin, atorvastatin, and lopinavir/ritonavir in a patient with HIV. AIDS Patient Care STDS 2003;17:207-10.

31. Castro JG, Gutierrez L. Rhabdomyolysis with acute renal failure probably related to the interaction of atorvastatin and delavirdine. Am J Med 2002;112: 505.

32. Aboulafia DM, Johnston R. Simvastatin-induced rhabdomyolysis in an HIV-infected patient with coronary artery disease. AIDS Patient Care STDS 2000; 14:13-8.

33. Martinez E, Tuset M, Milinkovic A, Miro JM, Gatell JM. Management of dyslipidemia in HIV-infected patients receiving antiretroviral therapy. Anitvir Ther 2004;9:649-63.

34. Barahona M, Mauri A, Sucunza N, Paredes R, Wagner AM. Hypothyroidism as a cause of rhabdomyolysis. Endocr J 2002;49:621-3.

35. Antons KA, Williams CD, Baker SK, Phillips PS. Clinical perspectives of statin-induced rhabdomyolysis. Am J Med 2006;119:400-9.

36. Pacerone (amiodarone) package insert. UpsherSmith Laboratories, Inc.; 2004. 